\title{
THE CONNECTIVITY FUNCTION OF A GRAPH
}

\section{LOWELL W. BEINEKE AND FRANK HARARY}

The vertex-connectivity and the edge-connectivity of a graph involve minimum sets of vertices and edges, respectively, whose removal results in a disconnected graph. However, the mixed case of separating sets consisting of both vertices and edges appears to have been overlooked. Such considerations might apply to vulnerability problems, such as that of disrupting a railway network with both tracks and depots being destroyed. Depending on the relative costs, a particular combination of tracks and depots might be optimal for the purpose.

The purpose of this note is to study the pairs $(k, l)$ of non-negative integers satisfying minimality conditions for disconnecting a graph by the removal of $k$ vertices and $l$ edges. It is shown that there are few restrictions on the collections of such pairs associated with some graph. The theorem of Menger on connectivity is then extended to the mixed cases of vertices and edges.

1. The connectivity function of a graph. The vertex-connectivity $\kappa$ (resp., edgeconnectivity $\lambda$ ) of a graph $G$ is the minimum number of vertices (resp., edges) whose removal results in a disconnected or trivial graph. The reason for the trivial graph being included in the definition is that there is no set of vertices whose removal disconnects a complete graph. One can also consider minimum sets of vertices or edges whose removal separates two specified vertices, called terminals, of a graph. The two " theories", which may be regarded as global and local, are equivalent, and we shall proceed from one to the other to suit the circumstances. In Fig. 1, for the terminals $s$ and $t$, the vertex-connectivity is 3 and the edge-connectivity 5 .

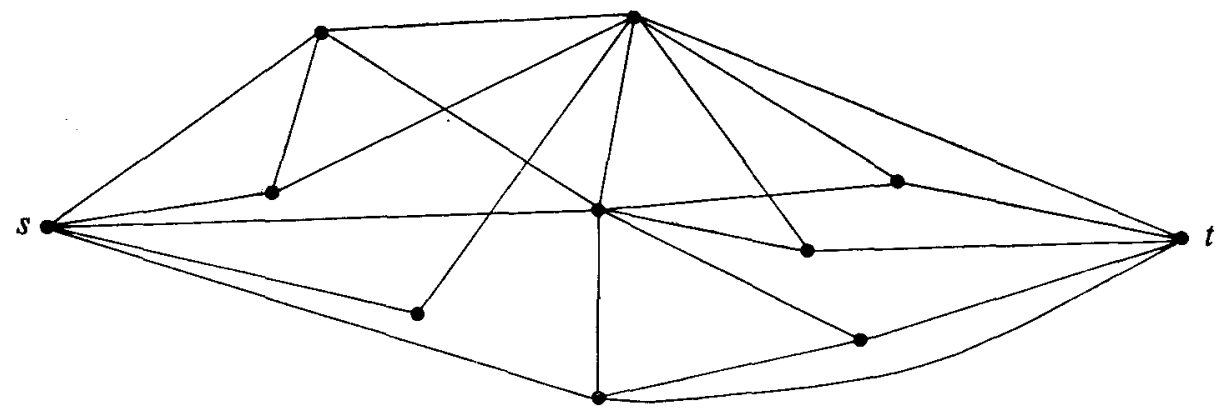

Fig. 1

A connectivity pair of a graph $G$ is an ordered pair $(k, l)$ of non-negative integers such that there is some set of $k$ vertices and $l$ edges whose removal disconnects the graph and there is no set of $k-1$ vertices and $l$ edges or of $k$ vertices and $l-1$ edges with this property. Clearly two connectivity pairs of $G$ are $(\kappa, 0)$ and $(0, \lambda)$, so that the vertex- and edge-connectivities are special cases of this concept. It is also readily seen that for each value of $k, 0 \leqslant k \leqslant \kappa$, there is a unique connectivity pair $\left(k, l_{k}\right)$; thus $G$ has exactly $\kappa+1$ connectivity pairs. An alternative (and equivalent) definition is that given $k \leqslant \kappa,(k, l)$ is a connectivity pair if $l$ is the minimum edge- 

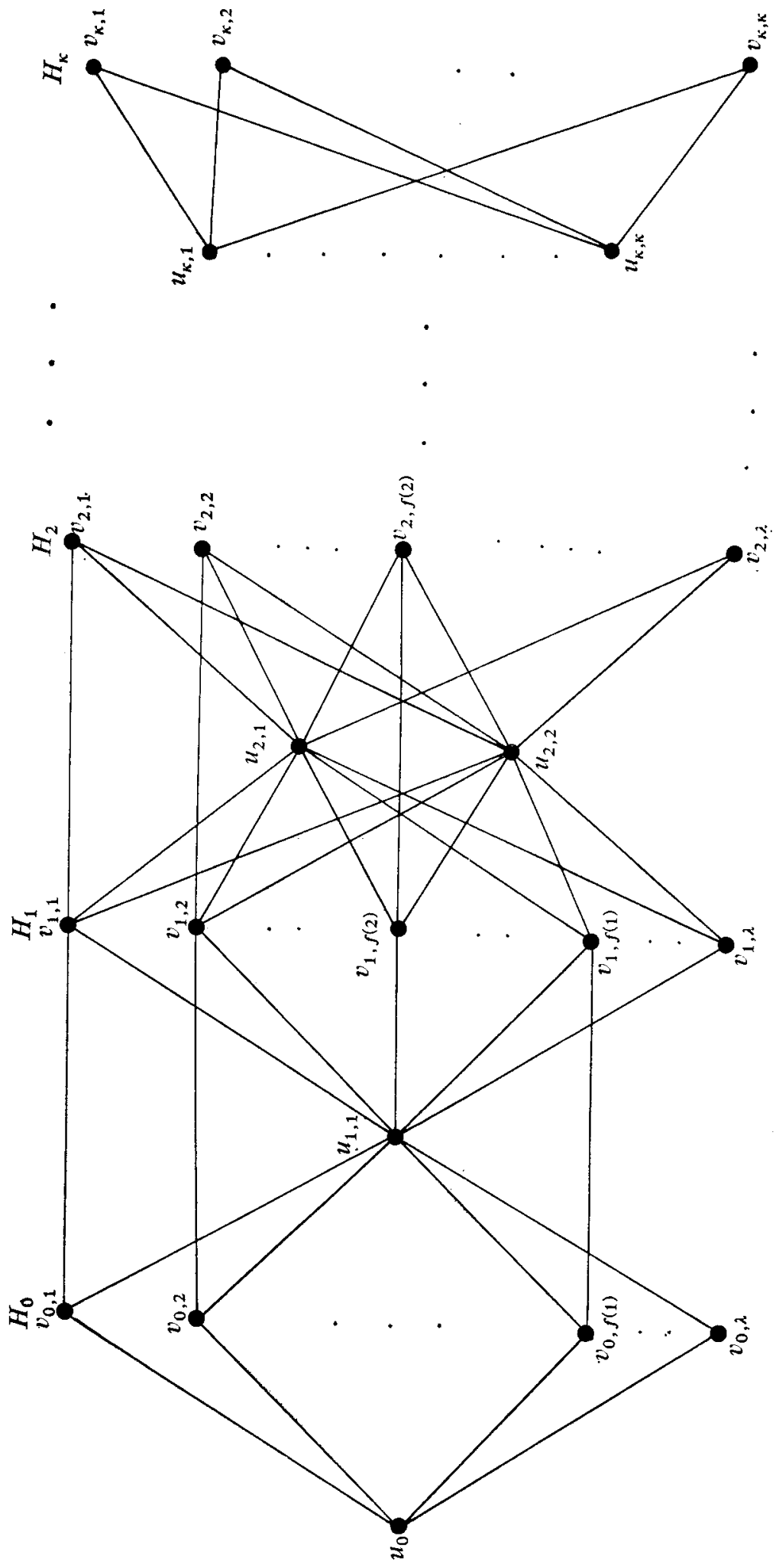

o 
connectivity among all subgraphs obtained by removing $k$ vertices from $G$. The graph $G$ of Fig. 1 has $(0,5),(1,4),(2,1)$, and $(3,0)$ as its connectivity pairs for the terminals $s$ and $t$.

The connectivity pairs of a graph thus determine a function $f$ from the set $\{0,1, \ldots, \kappa\}$ into the non-negative integers such that $f(\kappa)=0$. This is called the connectivity function of the graph. It is strictly decreasing, since if $(k, l)$ is a connectivity pair with $k<\kappa$ there is obviously a set of $k+1$ vertices and $l-1$ edges whose removal disconnects the graph or leaves only one vertex. The following theorem shows that these are the only conditions a connectivity function must satisfy.

THEOREM 1. Every decreasing function f from $\{0,1, \ldots, \kappa\}$ into the non-negative integers such that $f(\kappa)=0$ is the connectivity function of some graph.

All that is required to prove this theorem is a rather simple construction. Let $\lambda=f(0)$ and form $\kappa+1$ disjoint complete graphs $H_{0}, H_{1}, \ldots, H_{\kappa}$ each having $\lambda$ vertices. Denote the vertices of $H_{k}$ by $v_{k, j}$ for $j=1,2, \ldots, \lambda$. Add a vertex $u_{0}$ adjacent to every vertex in $H_{0}$. For $0<k \leqslant \kappa$, add vertices $u_{k, j}$ for $j=1,2, \ldots, k$ adjacent to all vertices in $H_{k-1}$ and $H_{k}$ and join vertices $v_{k-1, i}$ and $v_{k, i}$ for $i=1,2, \ldots, f(k)$. This determines a disconnecting set of $k$ vertices and $f(k)$ edges, for each $k$. That this has the minimality required for $(k, f(k))$ to be a connectivity pair is a bit tedious to verify, but not difficult. The details will be omitted.

Fig. 2 illustrates this construction. It is understood, but not shown, that $u_{3,1}$ is adjacent to all the vertices in $H_{2}$ and $H_{3}$, and so on.

This construction of course requires many vertices indeed. For two-terminal graphs one can give a construction requiring considerably fewer vertices, as follows. Take three sets, $U, V$, and $W$ of vertices with $U=\left\{u_{1}, u_{2}, \ldots, u_{\lambda}\right\}, V=\left\{v_{1}, v_{2}, \ldots, v_{\kappa}\right\}$ and $W=\left\{w_{1}, w_{2}, \ldots, w_{\lambda}\right\}$. Join one terminal $s$ to each vertex of $U$ and join the other terminal $t$ to each vertex of $W$. Make joins between $V$ and $W$ in the following way: For $0 \leqslant k \leqslant \kappa$, join $v_{j}$ to $w_{1}, w_{2}, \ldots, w_{f(k-1)}$ for each $j \leqslant k$. Make the corresponding joins between $U$ and $V$.

It is readily seen that the removal of the set of vertices $v_{1}, v_{2}, \ldots, v_{k}$ and edges $w_{1} t, w_{2} t, \ldots, w_{f(k)} t$ separates $s$ and $t$. For, if $j>k$, the vertex $v_{k}$ is adjacent only to vertices $w_{i}$ with $i \leqslant f(k)$, so there are no paths from $V$ to $t$ when the above set is removed.

Now assume that there is a set of $k$ vertices whose removal results in a graph $G^{\prime}$ in which fewer than $f(k)$ edges separate $s$ and $t$. Note that any vertex in $U, V$, or $W$ is adjacent to (at least) all those vertices to which a vertex in the same set but having a higher subscript is adjacent. Thus we may assume that the vertices of $V$ which have been removed are $v_{1}, v_{2}, \ldots, v_{m}(m<k)$. The vertex $v_{m+1}$ is adjacent to $w_{1}, w_{2}, \ldots, w_{f(m)}$ in $G$ and to at least all but $k-m$ of these vertices in $G^{\prime}$. Thus there are at least $f(m)-k+m$ paths of the form $v_{m+1} w_{j} t$ in $G^{\prime}$, and also this many paths of the form $s u_{j} v_{m+1}$. From the strictly decreasing property of $f$ it follows that $k-m \leqslant f(m)-f(k)$, so that there are at least $f(k)$ edge-disjoint paths $s u_{j} v_{m+1} w_{j} t$ joining $s$ and $t$ in $G^{\prime}$. This completes the proof that $(k, f(k))$ is indeed a connectivity pair.

This construction is illustrated in Fig. 3 for the pairs $(0,5),(1,3),(2,2),(3,0)$, where the pattern of differences in the second elements contrasts that of Fig. 1.

2. The mixed form of Menger's Theorem. The two variations of Menger's theorem 


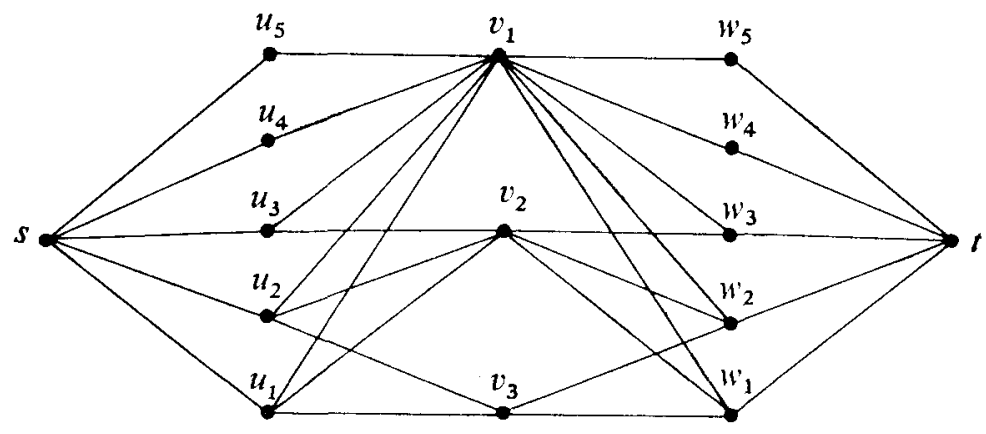

Fig. 3

pertaining to the vertex- and edge-connectivities of a graph are well-known. There are many equivalent ways of stating them, including the following.

THEOREM I. (Menger [4]). The maximum number of non-intersecting paths joining two non-adjacent vertices $s$ and $t$ is the minimum number of vertices whose removal separates $s$ and $t$.

Theorem II. (Elias, Feinstein, and Shannon [2]; Ford and Fulkerson [3]). The maximum number of edge-disjoint paths joining $s$ and $t$ is the minimum of edges whose removal separates them.

The two theorems above apply to two of the ordered pairs, namely $(\kappa, 0)$ and $(0, \lambda)$, of the connectivity function. The following theorem gives an extension to all of the connectivity pairs of the graph.

THEOREM 2. If $(k, l)$ is a connectivity pair for vertices $s$ and $t$ in graph $G$, then there are $k+l$ edge-disjoint paths joining $s$ and $t$, of which $k$ are mutually non-intersecting.

Before proving this result, some indication of its strength is given. Under the hypotheses of the theorem, there is of course a set of $k$ vertices and $l$ edges whose removal separates $s$ and $t$, so that any path joining them contains at least one of these $k+l$ elements. The theorem implies that one can choose $k+l$ edge-disjoint paths, each containing one element of the separating set. Those containing the vertices can be chosen to be mutually non-intersecting (a statement slightly stronger than the theorem). One might conjecture that this set of $k$ paths can be chosen so that each of them intersects none of the other $k+l-1$ paths. The graph in Fig. 4 shows that this is not always possible. One connectivity pair is $(2,2)$, but one cannot choose

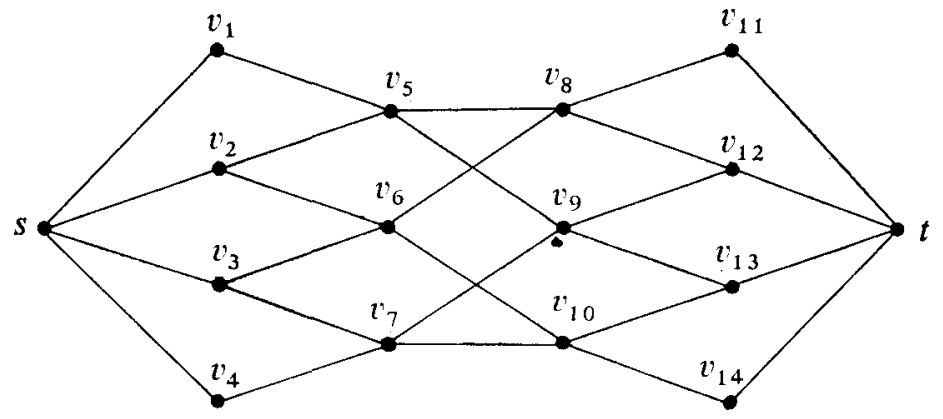

Fig. 4 
four edge-disjoint paths joining $s$ and $t$ so that two do not meet any of the others. Four paths which satisfy the theorem are

$$
s v_{1} v_{5} v_{8} v_{11} t, \quad s v_{2} v_{6} v_{10} v_{13} t, \quad s v_{3} v_{7} v_{9} v_{12} t, \quad s v_{4} v_{7} v_{10} v_{14} t .
$$

Here the vertices $v_{5}$ and $v_{6}$ and edges $v_{7} v_{9}$ and $v_{7} v_{10}$ separate $s$ and $t$, and the first two paths contain $v_{5}$ and $v_{6}$ and do not intersect.

Another conjecture which might be made is that the sets of $\lambda$ edge-disjoint paths and $\kappa$ non-intersecting paths joining two vertices can be chosen so that the latter are a subset of the former. Indeed, in the above example, the first three of the above four edge-disjoint paths are mutually non-intersecting. However, in the graph of Fig. 5, for the two terminals, $\kappa=3$ and $\lambda=5$, but any choice of three non-intersecting paths contains all of the edges $e_{i}$ of a cut set, so that no set can be extended to five-line disjoint paths.

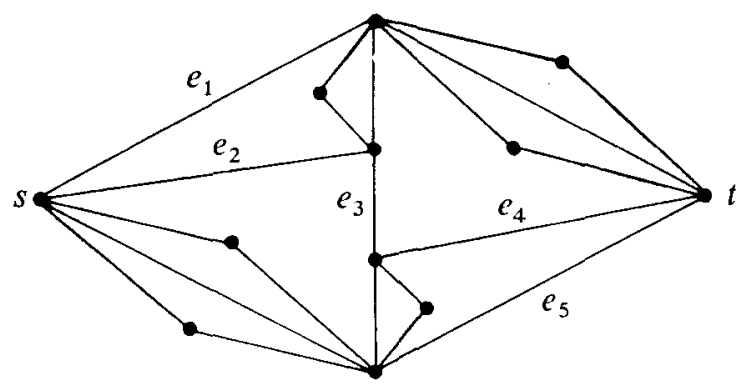

Fig. 5

Returning to Theorem 2 itself, the proof given here is based on that of Dirac [1] for Theorem I. Following his notation, an $s: t$ path is a path from $s$ to $t$, and a $(k, l)$-collection of $s: t$ paths consists of $k+l$ edge-disjoint $s: t$ paths of which $k$ are non-intersecting. Also, an $s: t$ connectivity pair and an $s: t$ separating set refer to the terminals $s$ and $t$ as expected. The proof is given for graphs which may have multiple edges joining pairs of vertices.

Proof. Assume the theorem is not true. Then there exists a graph with an $s: t$ connectivity pair $(k, l)$ which does not have a $(k, l)$-collection of $s: t$ paths. One can choose $k$ and $l$ so that $k+l$ is minimal in this respect. By Theorem II, we may also take $k>0$. Let $G$ be a graph with this property having the smallest number of vertices. Clearly, we can also assume that for any edge $e$ of $G,(k, l)$ is not an $s: t$ connectivity pair for $G-e$.

For any edge $e=u v$ of $G, G-e$ must have $(k, l-1)$ or $(k-1, l)$ as an $s: t$ connectivity pair. Let $A$ be a corresponding $s: t$ separating set. If $(k, l-1)$ is an $s: t$ connectivity pair of $G-e$, then $A \cup\{e\}$ is an $s: t$ separating set in $G$, while if $(k-1, l)$ is an $s: t$ connectivity pair of $G-e$, then $A \cup\{u\}$ is an $s: t$ separating set when $u \neq s$ or $t$ and $A \cup\{v\}$ is one when $v \neq s$ or $t$.

No vertex in an $s: t$ separating set can be adjacent to both $s$ and $t$. For, if $v$ is, then $G-v$ has $(k-1, l)$ as an $s: t$ connectivity pair and thus has a $(k-1, l)$-collection of $s: t$ paths by the minimality of $k+1$. These paths together with the path $s v t$ form a $(k, l)$-collection in $G$, which is impossible. Similarly, it is clear that no edge joins $s$ and $t$. 
Consider a mixed set $B$ of vertices and edges. We say that $B$ touches a vertex $w$ of $G$ if every element of $B$ is adjacent to or incident with $w$. We now show that every $(k, l)$ set $B$ which separates $s$ and $t$ touches $s$ or $t$. Assume the contrary and let $B=\left\{v_{1}, \ldots, v_{k}, e_{1}, \ldots, e_{l}\right\}$ with $e_{j}=s_{j} t_{j}$.

Let $G(s: B)$ be the subgraph of $G$ which is the union of all paths from $s$ to an element (vertex or edge) of $B$, containing no other element of $B$. Let $H$ be the graph obtained from $G(s: B)$ by identifying the $l$ vertices $t_{j}$ as $t^{\prime}$ and joining each $v_{i}$ to $t^{\prime}$. Then $H$ has fewer vertices than $G$ and has an $s: t^{\prime}$ separating set of $k$ vertices and $l$ edges; in fact, $\left\{v_{1}, \ldots, v_{k}, s_{1} t^{\prime}, \ldots, s_{l} t^{\prime}\right\}$ is such a set. Furthermore, $H$ cannot have an $s: t^{\prime}$ separating set of $k-1$ vertices and $l$ edges or $k$ vertices and $l-1$ edges since the corresponding set in $G$ would be an $s: t$ separating set, so that $(k, l)$ is an $s: t^{\prime}$ connectivity pair in $H$. Therefore there is in $H$ a $(k, l)$-collection of $s: t^{\prime}$ paths with those paths containing vertices $v_{i}$ being non-intersecting. These can be used to construct a corresponding collection of $k+l$ paths in $G(s: B)$, and hence in $G$, terminating in the elements of $B$, such that those containing any $v_{i}$ are non-intersecting. Similarly there is such a collection of $k+l$ paths in $G$ joining the elements of $B$ with $t$. These two collections of paths can be pieced together to get a $(k, l)$-collection of $s: t$ paths in $G$, an impossibility which verifies the assertion.

There must be a vertex $v$ not adjacent to both $s$ and $t$. Without loss, assume $v$ is at distance 2 from $s$, with suv being a path. Since $e=u v$ is not incident with $s$ or $t$, it cannot be in any $s: t$ separating set. Therefore, $(k-1, l)$ must be a connectivity pair for $G-e$. Let $A$ be a corresponding $s: t$ separating set as before. Then $A \cup\{u\}$ is a separating set in $G$, so that $u$ is in some separating set. Therefore $u$, being adjacent to $s$, is not adjacent to $t$. Hence, every element of $A \cup\{u\}$ is adjacent to or incident with $s$. But $A \cup\{v\}$ is also an $s: t$ separating set so that every vertex in it, in particular $v$, is also adjacent to $s$. But this contradicts the choice of $v$. The theorem is thus proved.

\section{References}

1. G. A. Dirac, " Short proof of Menger's graph theorem ", Mathematika, 13 (1966), 42-44.

2. P. Elias, A. Feinstein, and C. E. Shannon, "A note on the maximum flow through a network", IRE Trans. Inform. Theory, 2 (1956), 117-119.

3. L. R. Ford, Jr. and D. R. Fulkerson, "Maximum flow through a network", Canad. J. Math., 8 (1956), 399-404.

4. K. Menger, “Zur allgemeinen Kurventheorie ”, Fund. Math., 10 (1927), 96-115.

Purdue University, Fort Wayne.

University of Michigan, Ann Arbor.

(Received on the 27th of February, 1967.) 